\title{
Introducing Matters Arising
}

\author{
Discourse is fundamental to science. We now have a dedicated format for constructive comments on Nature \\ Methods papers, called Matters Arising.
}

$\mathrm{S}$ cientists before the internet age often discussed and debated ideas via lengthy handwritten correspondence with one another. A modern version of such discourse takes place on a multitude of online forums, including journal- or preprint-server-hosted commenting tools, initiatives such as PubPeer and Faculty of 1000 , community blogs, personal blogs and of course the omnipresent Twitter. Many journals, including Nature Methods, also have a formal route for publishing reader comments on papers. These comments are held to high editorial standards and are usually peer reviewed. This mechanism allows critiques to be linked directly to the paper in question and to become part of the scientific record.

About a year ago, following from Nature's announcement, we introduced Matters Arising as a new format dedicated to post-publication comments on Nature Methods papers. Matters Arising replaces the use of our Correspondence format for comments and responses (though we still maintain the Correspondence format for publishing announcements of tools, resources or initiatives of broad interest to our readership). Our first comment and response in this new format are published this month.

Matters Arising are intended as comments or critiques of papers published specifically in Nature Methods. If a manuscript highlights technical flaws or limitations in methodology used in multiple papers in the broader literature, supported by strong data and analysis, then it should be submitted as a Brief Communication. We also occasionally consider critiques of methodology that are more essay-like in nature; such a manuscript should be submitted as a Comment. If a manuscript describes a methodological development beyond what was previously reported in a published paper, then it should be submitted as an Article or Brief Communication.

Readers who find a problem with a Nature Methods paper should first try contacting the corresponding author; many misunderstandings can be resolved in such a manner, or errors can be fixed with a correction. If the corresponding author does not respond, or if their response does not resolve the issue, we then suggest that you contact us (methods@us.nature.com) and we will recommend whether it would be appropriate to submit a Matters Arising. More information on the editorial handling process for Matters Arising is available in our Guide to Authors.

As editors, we reserve the right to determine what we publish as Matters Arising. They will be held to the same strict editorial standards of interest, timeliness and quality to which we hold all of our publications. We will consider Matters Arising that raise serious issues central to the conclusions of the paper, such as regarding the utility of the method or the interpretations of the results. Matters Arising should be constructive and bring further clarity to a paper, such as by highlighting limitations or presenting alternative interpretations of the data. If there is a technical error in a paper that can be fixed, we would typically print an Author Correction or an Addendum rather than publish a Matters Arising. We do not consider omissions of references as cause for a Matters Arising; if a reader has identified potential missed citations, we (with the help of reviewers if necessary) will consider how central they are to the paper and in rare cases we may print a correction.

If we find that a Matters Arising submission has potential merit, we will invite the authors of the Nature Methods paper to write a response. Such a response is optional. Once we have this response, we will either reject the Matters Arising or send it and the response out for peer review. Following review, we will either invite revisions or reject one or both pieces. The Matters Arising authors will have the first opportunity to revise their comment in accordance with the response from the authors of the original paper and the reviewers' comments. The responding authors will then have the opportunity to revise their response in light of the updated Matters Arising, as well as the reviewers' comments. This process of review and revision continues until both pieces are ready for publication.

Because of the unique workflow for a Matters Arising, the editorial process can take quite some time. Matters Arising may raise complex technical issues that require new analyses on the part of the responding authors, extended review periods, and additional editorial discussions with reviewers. We do, however, recognize that there is a need to alert the broader research community of potential flaws before they propagate further, so we encourage Matters Arising authors to post a preprint of their work.

It should go without saying that personal attacks, strongly worded language and unjustified accusations of scientific fraud have no place in scholarly discourse - no matter whether one is doing the commenting or replying to the comment. Any Matters Arising that we publish will be edited carefully to ensure that the focus is solely on the science.

Criticism of one's work can feel personal and is never easy to take. We understand the tendency of authors to be defensive, or to try to brush the criticism off as trifling. Authors responding to a comment should keep the bigger picture in mind and be open to accepting and appropriately responding to critiques that can bring clarity, set the record straight, avoid propagation of errors, and help advance the field forward. Science is an ongoing process: peer review does not end with the publication of a paper, and there is always room for improvement.

It takes guts to call out flaws in a colleague's work, especially in a public forum, and we appreciate that some are willing to take the risk to improve the correctness of the scientific record. As always, we welcome your questions and your feedback about Matters Arising.

Published online: 4 March 2020 https://doi.org/10.1038/s41592-020-0791-2 